\title{
A prospective study of physician-observed concussion during a varsity university ice hockey season: incidence and neuropsychological changes. Part 2 of 4
}

\author{
Paul S. Echlin, M.D., ${ }^{1}$ Elaine N. Skopelja, M.A.L.S., ${ }^{2}$ Rachel Worsley, M.P.T., ${ }^{3}$ \\ Shiroy B. Dadachanji, M.D., ${ }^{4}$ D. Rob Lloyd-Smith, M.D., 5 \\ Jack A. Taunton, M.Sc., M.D., ${ }^{4}$ Lorie A. Forwell, M.Sc.P.T., ${ }^{6}$ \\ And Andrew M. Johnson, Ph.D. ${ }^{7}$
}

${ }^{I}$ Elliott Sports Medicine Clinic, Burlington, Ontario, Canada $;{ }^{2}$ School of Medicine Library, Indiana University, Indianapolis, Indiana; ${ }^{3}$ School of Physical Therapy, ${ }^{4}$ School of Kinesiology, and ${ }^{5}$ Department of Family Medicine, The University of British Columbia, Vancouver, British Columbia; ${ }^{6}$ Fowler Kennedy Sport Medicine Clinic and School of Physical Therapy, and ${ }^{7}$ School of Health Studies, The University of Western Ontario, London, Ontario, Canada

\begin{abstract}
Object. The primary objective of this study was to measure the incidence of concussion according to a relative number of athlete exposures among 25 male and 20 female varsity ice hockey players. The secondary objective was to present neuropsychological test results between preseason and postseason play and at 72 hours, 2 weeks, and 2 months after concussion.

Methods. Every player underwent baseline assessments using the Sport Concussion Assessment Tool-2 (SCAT2), Immediate Post-Concussion Assessment and Cognitive Test (ImPACT), and MRI. Each regular season and postseason game was observed by 2 independent observers (a physician and a nonphysician observer). Players with a diagnosed concussion were removed from the game, examined in the team physician's office using the SCAT2 and ImPACT, and sent to undergo MRI.

Results. Eleven concussions occurred during the 55 physician-observed games (20\%). The incidence of concussion, expressed as number of concussions per 1000 athlete exposures, was 10.70 for men and women combined in regular season play, 11.76 for men and women combined across both the regular season and playoff season, 7.50 for men and 14.93 for women in regular season play, and 8.47 for men across both the regular season and playoff season. One male player experienced repeat concussions. No concussions were reported during practice sessions, and 1 concussion was observed and diagnosed in an exhibition game. Neuropsychological testing suggested no statistically significant preseason/postseason differences between athletes who sustained a physician-diagnosed concussion and athletes who did not sustain a physician-diagnosed concussion on either the ImPACT or SCAT2. The athletes who sustained a physician-diagnosed concussion demonstrated few reliable changes postinjury.

Conclusions. Although the incidence of game-related concussions per 1000 athlete exposures in this study was half the highest rate reported in the authors' previous research, it was 3 times higher than the incidence reported by other authors within the literature concerning men's collegiate ice hockey and 5 times higher than the highest rate previously reported for woman's collegiate ice hockey. Interestingly, the present results suggest a substantively higher incidence of concussion among women (14.93) than men (7.50). The reproducible and significantly higher incidence of concussion among both men and woman ice hockey players, when compared with nonphysician-observed games, suggests a significant underestimation of sports concussion in the scientific literature.

(http://thejns.org/doi/abs/10.3171/2012.10.FOCUS12286)
\end{abstract}

$\begin{array}{lllll}\text { KeY WoRds } \bullet \text { concussion } & \bullet \text { ice hockey } \bullet & \text { incidence } \\ \text { neuropsychological testing } \bullet \text { sex } \bullet \text { Canada }\end{array}$

\footnotetext{
Abbreviations used in this paper: CIS = Canadian Interuniversity Sports; DTI = diffusion tensor imaging; HCEP = Hockey Concussion Education Project; ICC = intraclass correlation coefficient; ImPACT $=$ Immediate Post-Concussion Assessment and Cognitive Test; MBESS = Modified Balance Error Scoring System; MRS = magnetic resonance spectroscopy; NCAA $=$ National Collegiate Athletic Association; NHL = National Hockey League; SCAT2 $=$ Sport Concussion Assessment Tool-2; SWI = susceptibility weighted MR imaging.
}

PORTS concussions can have cumulative and longlasting effects on memory, judgment, social con$\checkmark$ duct, reflexes, speech, balance, and coordination. Research suggests an association between sports concussions and both immediate and later-life cognitive impairment. ${ }^{11,12,23,25,26,29,39,40,42}$ Data from a small sample of retired professional American football players suggest an increased risk and earlier onset of memory impairment, 
mild cognitive impairment, and Alzheimer's dementia., ${ }^{4,11,12}$ Recently, a large cohort mortality study of 3439 US National Football League players by Lehman et al. ${ }^{34}$ demonstrated that the neurodegenerative mortality rate of this cohort is 3 times higher than that of the general US population and that for 2 of the major neurodegenerative subcategories (Alzheimer disease and amyotrophic lateral sclerosis) the rate is 4 times higher.

The majority of research relating to sports concussion incidence has involved young men in high school, university, and professional sports, using retrospective reporting, retrospective analysis of injury databases, or prospective database analysis based on the reports of team medical staff. $13,14,24,27,28,33,41,43,44,54,56,57$ In 2010 , however, Echlin et al. ${ }^{16-19}$ reported on independent direct physician-observed concussions in junior ice hockey players. They found that the incidence of game-related concussions (per 1000 athlete exposures) for 2 fourth-tier junior ice hockey teams was 7 times higher than the highest rate previously reported in the literature (21.5 concussions per 1000 athlete exposures)..$^{18}$

The primary goal of this study was to prospectively measure the direct physician-observed and physician-diagnosed incidence of concussion (per 1000 athlete exposures) for 1 men's and 1 women's Canadian Interuniversity Sports (CIS) ice hockey team during 1 regular season and playoffs, using the concussion definition and return-toplay protocol from the 2009 Zurich consensus statement from the 3rd International Conference on Concussion in Sport.$^{38}$ Although the current diagnosis and return-to-play protocol for sports concussion is based on the presence of symptoms reported by the athlete and objective neuropsychological testing, there is insufficient literature that validates these widely used testing paradigms. Assessment methodologies are further complicated by the fact that symptom reporting by the athlete demonstrates significant variability and unreliability. This paper will examine the documented neuropsychological findings of the athletes in this study. Preseason and postseason testing (ImPACT and SCAT2) was performed on all participants, and concussed athletes were tested immediately after injury (SCAT2) and at postinjury intervals of 72 hours, 2 weeks, and 2 months (SCAT2 and ImPACT).

\section{Methods \\ Participants and Sampling}

The HCEP is a prospective cohort study that was conducted during 1 CIS ice hockey season (2011-2012). Between August 2011 and March 2012, approximately 45 CIS male and female university hockey players consented to participate in the HCEP. All players underwent comprehensive preseason baseline assessments involving neuropsychological testing (ImPACT and SCAT2) and 3-T MRI. Three types of MRI data were collected: DTI, MRS, and SWI. Magnetic resonance spectroscopy and DTI interpretation modalities are presented in subsequent papers within this issue. The SWI and women's team DTI results from this study will be presented in a future publication.

Individuals were excluded from participation in this study if they had a history of central neurological conditions other than head injury, severe cognitive impairment and/or an inability to consent, a history of pacemaker usage, previous eye surgery, or if they had worked in an environment that exposed them to a risk of having metal fragments embedded in their eyes. Each participant provided written informed consent and a release of medical information at the outset of the study. This study was approved by a university research ethics board.

\section{Outcome Assessment}

Two standardized concussion assessment tools, the SCAT2 and ImPACT, were administered to all players before the season began and to each player after a physician-observed and physician-diagnosed concussion. Although clinical assessment and judgment remain the gold standard in the diagnosis of concussions, neither the ImPACT nor the SCAT2 is supported by sufficiently rigorous scientific evidence to be used as standalone tools for medical decision making. These measures were used to evaluate individuals for the presence of possible cognitive deficits that have been associated with concussion.

The SCAT2 was developed at the 3rd International Conference on Concussion in Sport held in 2008 in $\mathrm{Zu}-$ rich, Switzerland, ${ }^{38}$ as an improved version of the original SCAT that was developed in 2005. ${ }^{37}$ The ImPACT is a computerized concussion evaluation system and is (at the time of this writing) the most widely used system for evaluating sports concussions, although it has not been independently evaluated. The ImPACT takes approximately 20 minutes to complete and measures multiple aspects of cognitive functioning in athletes including attention span, working memory, sustained and selective attention time, response variability, and reaction time.

\section{Study Procedure}

At the ice hockey rink the SCAT2-including the Symptom Inventory, Glasgow Coma Scale, Maddocks Score, Coordination Examination, MBESS, and Standardized Assessment of Concussion-was recorded during concussion evaluation by a physician. The SCAT2 utilizes the MBESS that is performed on the ground and does not use a secondary balance platform for the 3 balance positions. At the postinjury office site the clinical evaluation and the ImPACT and SCAT2 evaluations (except for the Maddocks Score) were repeated.

The ImPACT results for the HCEP were independently evaluated by a remote neuropsychologist. Although the ImPACT is a commonly used and relatively accessible test, it is a psychometric test that calls for a refined level of understanding available to the registered psychologist with training in psychometrics and neuropsychological diagnostic skills..$^{50}$ The remote independent neuropsychological analysis of this diagnostic tool also eliminates possible bias that may exist when a team physician is interpreting the test for an athlete.

Concussion and Self-Report Definitions. Concussion was clinically diagnosed using an observed or self-reported mechanism (such as a blow to the head or body) and immediate or delayed neurological signs (for 
example, a player is unstable, lacks coordination, or is slow to return to the play) or symptoms (such as a player experiences headache, dizziness, or alteration of vision). Abnormal SCAT2 or ImPACT results were also used to guide diagnosis. A self-reported concussion was defined as a concussion that was not identified by the physician or nonphysician observers at an observed game. These players subsequently presented to the physician for diagnostic evaluation, either at the end of the game or in the days following that game. A flowchart concerning the methods of concussion identification, treatment, and return-to-play protocol is provided (Fig. 1).

Concussion Surveillance, Identification, and Diagnosis. Concussion surveillance was conducted at each preseason, regular season, and postseason game by 2 independent observers: 1 physician and 1 nonphysician. Independent observers were defined as individuals who were not affiliated on a regular basis with the teams that they were evaluating. This independence allows for the elimination of possible bias that may affect the evaluation and return-to-play decision due to familiarity with the athletes and the team. Each observer was asked to complete a 23-question descriptive form to document each observed and diagnosed concussion.

Observers were placed at elevated and well-balanced positions around the ice rink to have direct views of play. Practice sessions were not directly observed during this study. For all observed concussions, the trainer was asked by the physician to remove the player from the game. The player was then to be immediately evaluated by the physician, away from the dressing room and ice surface, while the other observer remained in place. Clinical evaluations of the player with a suspected concussion included SCAT2 evaluation at the rink.

Clinical Return-to-Play Definition. The return-toplay decision during a game is made by the HCEP physician after an evaluation of the individual with a suspected concussion is completed and is negative. All return-toplay decisions after a diagnosed concussion is made should be made by the team physician. The team physician has access to the results of the SCAT2 and ImPACT scores (along with interpretation by a neuropsychologist) and MRI. After clinical clearance was obtained according to the Zurich protocol, each injured player was returned to competitive play.

\section{Statistical Analysis}

The incidence of concussion was calculated as the number of physician-observed or self-reported concussions occurring during physician-observed games, divided by the total number of athlete exposures. Athlete exposures were defined within this sample as the sum of all "games played" and were summed for all players. This method of determining the denominator for the incidence calculation was deliberately conservative and included all athlete exposures, regardless of the length of time played within any given game.

Exhibition games were not used in incidence calculations because official game sheets were not available to the investigators to establish the number of games played.

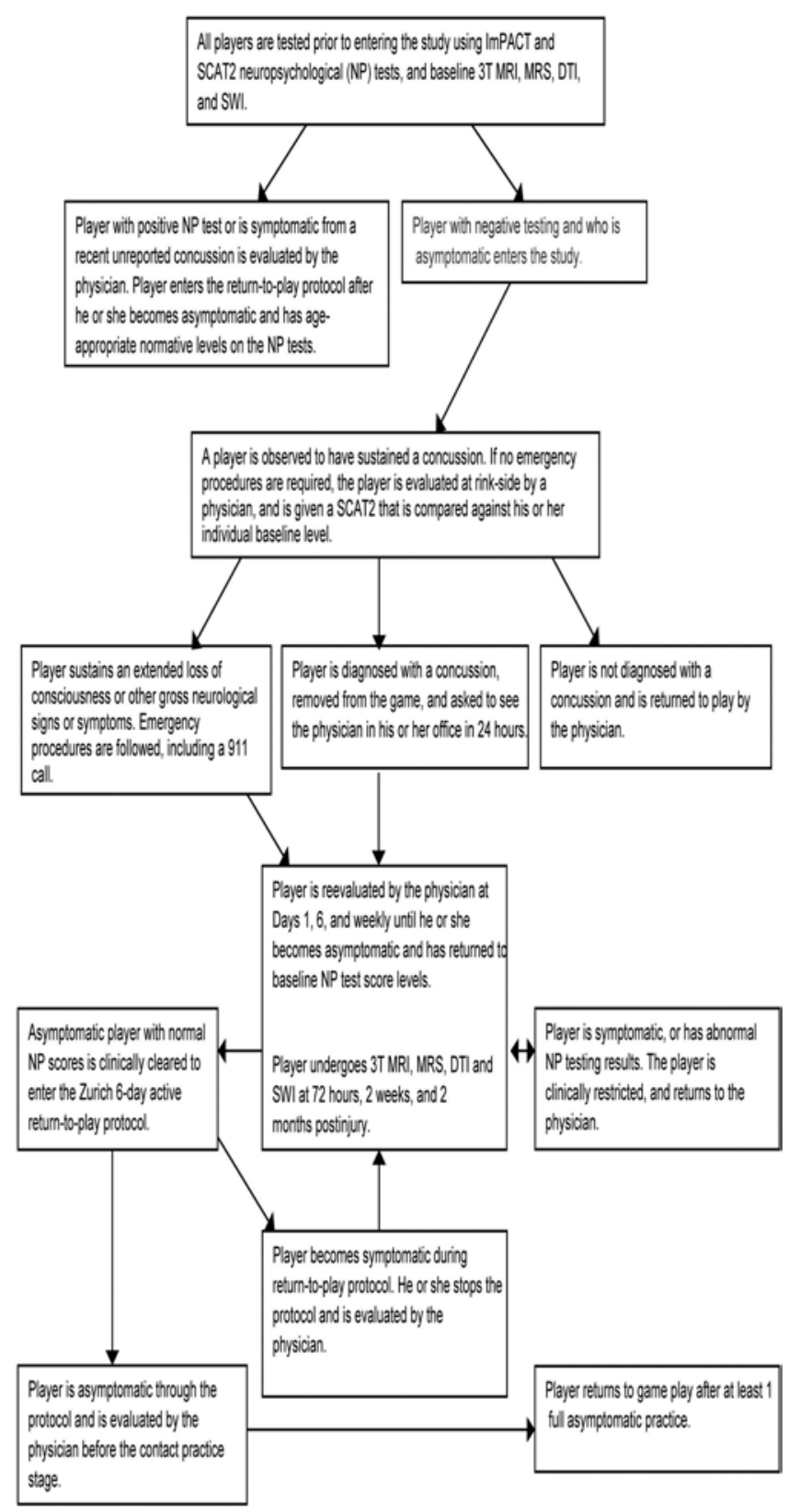

FIG. 1. Flowchart describing the experimental protocol.

Goalie incidence of concussion was calculated from standardized game sheets. If a goalie dressed for a game but did not play, that game was not used in the incidence calculation.

Test-retest reliability for both the ImPACT and SCAT2 was calculated using 2-way random effects single-measure ICCs (ICC[2,1]). The neuropsychological data (ImPACT and SCAT2 results) were then analyzed separately using repeated-measures multivariate ANOVA calculations. Preseason and postseason function was compared between concussed and nonconcussed players using a $2 \times 2$ (concussion status by time period) multivariate ANOVA. Subsequently, preseason function was compared with the 3 postinjury time periods ( 72 hours, 2 weeks, and 2 months). Statistically significant univariate findings were evaluated 
using reverse Helmert contrasts (the mean of each level was compared with the mean of the previous levels). Five scales of the ImPACT (verbal memory composite, visual memory composite, motor composite, reaction time composite, and impulse control) and 4 scales of the SCAT2 (number of symptoms, physical symptoms, balance, and coordination) were evaluated within these analyses. All the individual scales of the SCAT2 test were analyzed, but only the total symptom score and the MBESS scores are presented in the tables. None of the other scales demonstrated a significant change preseason and postseason or after injury.

\section{Results}

\section{Incidence Calculations}

The men's team played 28 CIS regular season games in 2011-2012 and 3 CIS playoff games. Concussion incidence was calculated separately for the men and the women. Among the men, 4 concussions were observed over the course of the regular season (533 athlete exposures), yielding a concussion rate of 7.50 concussions per 1000 athlete exposures, and 8.47 per 1000 athlete exposures for the regular and playoff games combined (590 athlete exposures). Five $(20 \%)$ of the 25 players who started the 2011-2012 regular season sustained at least 1 concussion. Sixteen percent (5 of 31) of the men's team games (including playoff games) involved at least 1 concussion. No male players with a concussion required hospital transfer and observation.

The woman's team played 24 regular season CIS games and no playoff games. Among women, 6 concussions were observed over the course of the season (402 athlete exposures), yielding a concussion rate of 14.93 concussions per 1000 athlete exposures. Twenty-five percent of the games played involved at least 1 concussion. Six $(28.6 \%)$ of the 21 individuals on the women's team who started the 2011-2012 regular season sustained at least 1 concussion. No concussed female players required hospital transfer.

Eleven concussions (20\%) occurred during the 55 physician-observed games. The combined male and female regular season incidence was 10.70 concussions per 1000 athlete exposures, and combined male and female regular and playoff incidence was 11.76 (992 athlete exposures).

Although not part of the incidence calculations, information on prior concussions was collected on participants. Fourteen (56\%) of the 25 men's team players admitted that they had suffered at least 1 concussion prior to the start of this study. All (100\%) of the 5 male players who had suffered a concussion diagnosed in the study had also reported a prior history of at least 1 concussion (3 of 5 had suffered 2 or more concussions). Four (20\%) of the 20 women's team players admitted that they had suffered at least 1 concussion prior to the start of the study. Three $(50 \%)$ of the 6 women who suffered a concussion during the study had reported a prior history of at least 1 concussion.

\section{Baseline Testing}

Seven ImPACT and 4 SCAT2 results were found to be abnormal after review by the remote neuropsychologist. These tests were repeated to determine if the athlete was injured when entering the study or if the results were anomalous for the individual. On repeat testing and clinical evaluation, none of the individuals were considered to have a current concussion entering the study.

\section{Neuropsychological Testing}

Reliability calculations are presented in Table 1 for both the ImPACT and the SCAT2. Reliability of these measures ranged from 0.075 to 0.651 , suggesting that there was a substantial amount of error associated with these neuropsychological test batteries (that is, the scores were not stable over time). All reliability calculations were conducted using only those participants who did not sustain a physician-observed concussion during play.

Descriptive statistics for the preseason/postseason comparisons between individuals who suffered a physician-diagnosed concussion and individuals who did not sustain a physician-observed concussion are presented in Table 2 for both the ImPACT and SCAT2, and descriptive statistics for the postinjury data (among concussed athletes) are presented in Table 3 for both of these tests.

Within the ImPACT, no significant interaction effects were demonstrated between the concussion variable and the time variable, suggesting that the ImPACT results were not significantly different between the preseason and postseason time periods. The multivariate test for the postinjury analysis among the concussed athletes was not significant $\left(\mathrm{F}_{(15,39)}=1.50\right)$, and among the univariate analyses, only the memory composite (verbal) showed a significant change over time $\left(\mathrm{F}_{(3,15)}=4.08, \mathrm{p}<0.05\right)$. Post hoc analysis suggested that the change between baseline and 72 hours was statistically significant $\left(\mathrm{F}_{(1,5)}=8.022, \mathrm{p}\right.$ $<0.05)$, but that no other changes were statistically significant at an $\alpha$ of 0.05 . Counterintuitively, the ImPACT score appeared to increase (rather than decrease) between baseline and 72 hours.

Two scales of the SCAT2 (physical signs and the coordination subscale) demonstrated insufficient variability to be analyzed within either of the multivariate ANOVAs, and so the analysis was run without these variables included in the design. Within the SCAT2, no significant inter-

TABLE 1: Test-retest reliability information for selected scales on the ImPACT and SCAT2

\begin{tabular}{ll}
\hline \multicolumn{1}{c}{ Variable } & ICC \\
\hline ImPACT & \\
memory composite (verbal) & 0.147 \\
memory composite (visual) & 0.389 \\
visual motor speed composite & 0.619 \\
reaction time composite & 0.546 \\
impulse control & 0.651 \\
total no. of symptoms & 0.335 \\
SCAT2 & \\
total symptom score & 0.445 \\
MBESS & 0.075 \\
\hline
\end{tabular}


Ice hockey concussion incidence and neuropsychological changes

TABLE 2: Scores on the ImPACT and SCAT2 among both nonconcussed and concussed athletes measured before and after the season*

\begin{tabular}{|c|c|c|c|c|}
\hline \multirow[b]{2}{*}{ Variable } & \multicolumn{2}{|c|}{ Nonconcussed } & \multicolumn{2}{|c|}{ Concussed } \\
\hline & Preseason & Postseason & Preseason & Postseason \\
\hline \multicolumn{5}{|l|}{ ImPACT } \\
\hline total symptom score & $3.22(5.09)$ & $3.47(4.94)$ & $7.10(6.67)$ & $4.70(8.59)$ \\
\hline memory composite (verbal) & $88.28(10.64)$ & $91.25(7.89)$ & $84.10(11.43)$ & $91.30(6.95)$ \\
\hline memory composite (visual) & $80.28(12.68)$ & $78.91(12.84)$ & $73.70(16.65)$ & $79.70(14.27)$ \\
\hline visual motor speed composite & $41.38(6.44)$ & $44.48(5.58)$ & $39.47(6.98)$ & $42.89(8.35)$ \\
\hline reaction time composite & $0.56(0.07)$ & $0.54(0.07)$ & $0.58(0.05)$ & $0.57(0.07)$ \\
\hline impulse control composite & $3.88(2.66)$ & $4.44(3.56)$ & $8.20(10.69)$ & $7.80(4.26)$ \\
\hline \multicolumn{5}{|l|}{ SCAT2 } \\
\hline total symptom score $†$ & $20.33(2.42)$ & $20.48(2.00)$ & $18.82(3.09)$ & $19.36(3.23)$ \\
\hline MBESS & $25.15(5.36)$ & $27.52(1.86)$ & $25.92(2.88)$ & $26.36(2.87)$ \\
\hline
\end{tabular}

action effects were demonstrated between the concussion variable and the time variable, suggesting that the SCAT2 was not significantly different between the preseason and postseason time periods. However, the multivariate test for the postinjury analysis among the concussed athletes was significant $\left(\mathrm{F}_{(8,64)}=2.26, \mathrm{p}<0.05\right)$, and the univariate analysis of the total symptom score was statistically significant $\left(\mathrm{F}_{(4,32)}=5.71, \mathrm{p}<0.05\right)$. Post hoc analysis of this significant omnibus effect demonstrated a significant change between 2 weeks postinjury and 2 months postinjury $\left(\mathrm{F}_{(1,8)}=17.56, \mathrm{p}<0.05\right)$, and between 2 months postinjury and the postseason evaluation $\left(\mathrm{F}_{(1,8)}=15.32, \mathrm{p}\right.$ $<0.05)$. The number of symptoms increased immediately after the injury and then returned to baseline values by the time of the postseason evaluation.

\section{Return to Play}

Of the 5 men who were identified to have suffered concussions during this study, 3 did not miss any games and were returned to play in less than 7 days. One of these 3 players sustained an observed and probable concussion prior to his first diagnosed concussion, but it was not clin- ically diagnosed. This player also refused to participate in further testing after his first set of postinjury evaluations.

Two players on the men's team with diagnosed concussions missed games secondary to their injury. One player did not play for the rest of the season due to ongoing postconcussion symptoms and missed 18 regular season games and 3 playoff games. The other player was injured in the first playoff game and subsequently did not play the final 2 playoff games. The woman's team had 2 players who sustained concussions and did not miss any games. One of these players was identified, but not diagnosed, until she self-presented after the end of the season. The other player returned to play after completing the 6-step return-to-play protocol.

Three of the women who sustained a concussion had to end their seasons early (missing 6, 6, and 8 games, respectively). One of these players attempted to return to play after missing 3 games (50 days), but could not play due to her ongoing symptoms after that game, and missed the final 5 games of the season. This unsuccessful return could have been due to a second (unobserved) concussion or to an incomplete recovery period.

TABLE 3: Scores on the ImPACT and SCAT2 among concussed athletes measured at 5 different time points*

\begin{tabular}{lccccc}
\hline \multicolumn{1}{c}{ Variable } & Preseason & 72 Hrs & 2 Wks & 2 Mos & Postseason \\
\hline ImPACT & & & & & \\
$\quad$ memory composite (verbal) & $83.33(9.67)$ & $93.6(7.63)$ & $86.83(9.89)$ & $93.33(6.22)$ & $91.50(6.86)$ \\
memory composite (visual) & $78.33(5.99)$ & $85.33(14.68)$ & $78.33(20.77)$ & $83.83(13.76)$ & $83.50(10.82)$ \\
visual motor speed composite & $41.71(7.19)$ & $44.59(7.21)$ & $44.30(7.00)$ & $46.47(4.13)$ & $48.31(4.68)$ \\
$\quad$ reaction time composite & $0.58(0.05)$ & $0.58(0.08)$ & $0.59(0.13)$ & $0.59(0.08)$ & $0.56(0.07)$ \\
$\quad$ impulse control composite & $3.83(2.56)$ & $4.00(2.45)$ & $4.17(2.23)$ & $6.67(2.50)$ & $6.83(3.76)$ \\
SCAT2 & & & & & \\
$\quad$ total symptom score $\dagger$ & $18.22(3.11)$ & $13.67(6.50)$ & $14.33(6.80)$ & $20.56(2.01)$ & $19.89(2.26)$ \\
$\quad$ MBESS & $26.89(1.97)$ & $25.44(4.13)$ & $26.11(4.57)$ & $26.56(3.43)$ & $26.33(3.12)$ \\
\hline * All data given as mean (SD). & & & &
\end{tabular}




\section{P. S. Echlin et al.}

\section{Descriptive Data on Concussed Players}

Only 1 player on the men's team received a major penalty for fighting and an automatic game misconduct and ejection from the game, as per the CIS rules concerning fighting. This individual was not examined for a concussion. None of the women were assessed a major/game misconduct for fighting.

Previous research ${ }^{18}$ presents evidence to suggest that $24 \%$ (5 of 21) of the diagnosed concussions that were observed resulted from direct involvement in a fight. In this 2011-2012 study only 1 fight was recorded to have occurred during the men's season. No concussions were reported to have resulted from that fight. This difference may demonstrate the fact that a significant penalty (minor penalty and a game misconduct) given to combatants in the CIS for fighting may discourage individuals from fighting, and thus may decrease this cause of concussion. This level of penalty did not exist in the league hosting the 2009-2010 teams.

During the 2011-2012 HCEP study, 11 concussions (5 in men and 6 in women) were diagnosed in 55 physicianobserved games. One concussion was observed and diagnosed in a preseason exhibition game. Observed preseason concussions were not included in the incidence calculations due to the lack of accurate player participation data during those games. Thirteen physician-observer evaluations were performed that resulted in a negative diagnosis, and the player was returned to play in the same game.

The observer reports concerning the 12 diagnosed concussions, including the 1 physician-observed preseason concussion that was not included in the incidence statistics, found that: 1) 9 of 11 individuals were forwards, 2 were defensemen, and none were goalies; 2) 8 concussions were diagnosed immediately, and these players were restricted from further play; 3$) 3$ concussions were self-reported due to delayed onset of perceived symptoms (1 player overtly avoided telling the physician so that he could continue playing); 4) 2 players (1 male and 1 female) with medically diagnosed concussions were allowed to continue playing against direct medical advice; 5) the contact that was observed to cause the concussion was to the head in 8 of 12 concussions ( 5 women and 3 men) and to the body in 4 of 12 concussions ( 1 woman and 3 men); 6) the contact that was believed to have caused the concussion was judged to be purposeful in 10 of 12 concussions ( 5 men and 5 women) and accidental in 2 of 12 concussions ( 1 elbow to the head and 1 hit to the body); and 7) 4 of the concussions occurred in the first period, 4 occurred in the second period, 1 occurred in the third period, and the timing of 3 concussions was not determined due to self-report presentation.

\section{Discussion}

The literature reports the incidence of concussion in terms of level of play, age, and sex of participants. The most common denominators for incidence calculation are player hours, athlete exposures, and games played. ${ }^{1-3,5,6,20-22,53}$ Flik et al. ${ }^{22}$ reported an incidence of concussion for Division I university ice hockey players of 3.1 per 1000 athlete exposures in 1 season. The incidence of concussion (per 1000 athlete exposures) in the present study was 3 times higher than the highest rate reported by these authors.

A 6-year study of the incidence of all injures (including concussion) that were diagnosed by the team physician of a CIS men's hockey team was conducted by Rishiraj et al., ${ }^{47}$ and the incidence of concussion was reported to be 1.7 per 1000 athlete exposures. In this study no concussions were identified in practice sessions.

Agel et al. ${ }^{2}$ documented a 16-year review of NCAA men's ice hockey injuries, and found an incidence of concussion of 1.47 per 1000 athlete exposures. This information was gathered from a retrospective review of the NCAA injury surveillance data for that period that had been entered by the team therapist. In a similar study, Agel et al. ${ }^{1}$ documented a 4-year review of the incidence of concussion among female ice hockey players, finding that concussions were the most common injury in both games and practices, with an incidence of 2.72 per 1000 athlete exposures in games. A 7-year review of women's and men's US NCAA college hockey by Agel and Harvey ${ }^{3}$ found that over that period the men's teams had an incidence of concussion of 0.72 per 1000 athlete exposures compared with 0.82 per 1000 athlete exposures for the women. These injuries were not independently observed and were part of a retrospective database review.

Wennberg and Tator ${ }^{53}$ reported an incidence of concussion in the NHL that ranged from 1.81 per 1000 athlete exposures in 1998-1999 to a low of 1.04 per 1000 athlete exposures in 2005-2006. A published study supported by the NHL in 2011 demonstrated that 559 physician-diagnosed concussions had been recorded in the 7-year period between 1997-1998 and 2003-2004. The incidence of concussion reported by these authors was 1.8 per 1000 player hours. ${ }^{5}$ A conservative calculated incidence of this study would be 1.4 per 1000 athlete exposures, assuming that all players played in all their games.

The incidence of concussion for female collegiate ice hockey players found in our study is 5.5 times greater than the rate found by Agel et al. in $2007^{1}$ and 18.2 times greater than the rate found by Agel et al. in 2010. ${ }^{3}$ Our 2011-2012 rates of concussions observed per game for the men's (19\%) and women's teams (25\%) were far higher than the rate previously reported by the NHL, which was a game rate of $3.1 \%$ reported in $2008 .^{32}$

In this study there was a significant difference in concussion incidence rate compared with previous rates in the literature. There are several possible reasons for this significant difference. First, other than our own previous research, ${ }^{16-19}$ other studies have not been exclusively dependent upon prospective, direct, physician-based concussion identification, diagnosis, and follow-up. The majority of studies depend on either the retrospective self-reporting of the athlete or the judgment of a certified team trainer or first responder. ${ }^{13,14,24,27,28,33,41,43,44,54,56,57}$ Second, the reluctance to report a concussion most commonly occurs because the athlete is either unaware of the seriousness of this brain injury or fears that he or she will be restricted from play.9,10,31,36,51 Kaut et al. ${ }^{31}$ found that $30.4 \%$ of athletes admitted to continuing to play while experiencing symptoms after being hit in the head, with 


\section{Ice hockey concussion incidence and neuropsychological changes}

football players exhibiting a high incidence of this behavior $(61.2 \%)$. Only $43 \%$ of all athletes surveyed stated they had some knowledge of concussions.

The independent observer reports were essential to providing a technical and social background to the data collected in this study. These reports also provided an unbiased depiction of the collection methods and mechanism of injury. As was the case in our previous research, ${ }^{18}$ the observer reports suggest that the majority of concussions resulted from direct hits to the head, and the majority of these hits were also described as purposeful. There were 3 of 11 concussions that were diagnosed due to selfpresentation to the physician following a later development of symptoms. One of these 3 individuals attempted to overtly conceal her symptoms from the physician to play another game. Two players (1 male and 1 female) returned to play against medical advice within the same game in which they had been diagnosed with a concussion. Interestingly, despite access to an experienced neuropsychologist to evaluate the objective test results, the services of this specialist were only used for 4 players.

An unpublished 2010-2011 study that we undertook after our 2009-2010 research involved a variant of our published methodology. ${ }^{18}$ In this study, a CIS men's varsity hockey team underwent preseason neuropsychological testing using the SCAT2 and ImPACT. The players, coaching staff, training staff, medical staff, and athletic department were made aware of the purpose and methodology of the study. The players were all exposed to concussion education material before the start of their hockey season. The medical and training staffs were requested to follow the Zurich guidelines for injury identification, postinjury testing, and return to play.

Unlike the initial 2009-2010 study, there were no independent physician and nonphysician observers present at each game. Despite the specific education involved, only 1 concussion was identified in the regular season $(2$ concussions were diagnosed in the preseason). Even allowing for the possibility of sample-specific variability, this incidence is quite low when compared with the current study, in which 4 regular season concussions were identified among the men and 6 regular season concussions were identified among the women. This rate is even lower than our published study (based on the 2009-2010 data), ${ }^{18}$ in which 1 of the teams had 13 diagnosed concussions during the regular season.

This disparity in the incidence of concussion may demonstrate the reluctance of individuals to self-report, or it may illustrate the game physician's reluctance to directly intervene, observe, and evaluate players that he or she suspects of having suffered a concussion. There may have been a learning curve for the specialty-trained sports physicians who were asked to identify, diagnose, and restrict the players during game action in an environment in which the coach or therapist traditionally fulfilled this role.

It is also possible that the incidence of concussion may depend (in part) on the level of experience of the physicians involved. In our previously published research ${ }^{18}$ we used 7 physician observers, including the lead HCEP physician. The lead HCEP physician observed 38 games, and the remaining 5 physicians observed a total of 14 games $(6,4,2,1$, and 1 , respectively). In the present research, 14 different physicians were employed, and the task of observing was more evenly distributed: the lead physician observed 14 games, 1 physician observed 13 games, 5 physicians observed 4 games each, and 4 physicians observed 2 games. In the unpublished 2010-2011 study, 5 team physicians were used, with 1 designated head team physician covering 10 games, and the other assisting team physicians covering $3,2,2$, and 1 game, respectively. Away games were covered by the team trainer only, and not the physicians involved in the study. There was no observational learning curve as the participating team physicians were not requested to specifically observe, identify, and diagnose players with a suspected concussion.

The higher incidence of concussion in direct observation among the sports medicine physicians in the initial study may have resulted from the accumulated focused and direct observation experience of 1 physician who covered the majority of the games, as compared with our other research in which the individual physicians acquired less experience in identifying hockey concussions (and will have less experience dealing with the social aspects of concussion diagnosis), due to the fact that they observe fewer games. In clinical practice, the physician is usually presented with an injured athlete who requires and appreciates his or her expertise. In this study, the injured athletes presented with verbalized suspicion and mistrust of these independent physicians, due to player beliefs that the physicians would restrict the player unnecessarily.

The elevated and multisite points of observation provided an improved ability to view the events occurring on the ice surface. The observers in this study were specifically looking for concussions prospectively, as opposed to collecting data retrospectively or using injury data previously collected. The observers in this current study likely observed and recorded more events than otherwise might have been detected by team staff who have a multitude of other duties. During the exit interviews of the observers, the majority of their responses positively favored the elevated position to observe a greater amount of the ice surface to look for an occurrence of a concussion, due to the speed of the game. We did not specifically monitor team practices, and no concussions that occurred in practices were reported by either team during the study. Previous sports concussion studies have demonstrated that concussion predominantly occurs in game situations., ${ }^{1,2,6,22,55}$

The return-to-play interval after a concussion is an important factor to prevent recurrent concussions. Guskiewitz et al. ${ }^{27}$ have demonstrated that $75 \%$ of recurrent concussions occurred within 7 days of the original injury, and $95 \%$ of these injuries occurred within 10 days. Echlin et al. ${ }^{18}$ reported that direct and independent observation combined with a rigorous return-to-play definition produced a recurrence rate of $28 \%$. In this 2010 study, ${ }_{18}^{18}$ for athletes using the 6-step Zurich return-to-play protocol, the mean interval between the first and second concussions was 78.6 days. The only repeat concussion, which occurred during this current study, was to a male 


\section{P. S. Echlin et al.}

player with an injury interval of 48 days. A possible repeat concussion may have occurred with 1 of the female players who attempted to return to play after missing 3 games over an interval of 50 days. Although she was not observed to have sustained a repeat concussion, her symptoms returned after her return game, and she missed the final 5 games of the season. This result could have been due to a second concussion or an incomplete recovery period.

Echlin et al. ${ }^{18}$ also reported that $42 \%$ of all participants who provided a concussion history admitted to suffering a concussion prior to the study, as compared with $45 \%$ of all participants in this current study $(56 \%$ of men and $20 \%$ of women). Furthermore, $88 \%$ (15 of 17) of those who suffered a concussion admitted to suffering at least 1 concussion in the past, compared with 55\% (6 of 11) in the current study. Although there are some variants in competition level (university vs fourth-tier junior men's ice hockey, and sex differences), there is a significant commonality in that those individuals who suffered an observed concussion were more likely to have had a history of prior concussions. In addition, the effect of number of concussions previously suffered demonstrates a strong trend for reinjury. In this study all 3 athletes who had admitted to 3 or more prior concussions also suffered one within this study. These trends are noted in the current literature concerning recurrent concussions..$^{24,28,57}$

Multisport studies in the literature have demonstrated a sex difference trend in concussion incidence. In an early review of multiple NCAA sports, Covassin et al. ${ }^{8}$ reported that women sustained a higher incidence of concussion across different sports. Covassin et al. ${ }^{7}$ have also recently reported sex differences among high school and collegiate athletes who have incurred a sports-related concussion.

Dick $^{15}$ reviewed the past 10 years of literature concerning sex differences in concussion in comparable sports. He found that female athletes may be at greater risk for concussion than their male counterparts. Although the evidence concerning sex differences in concussion is currently lacking, the reasons that these authors gave involved 3 different areas of study that are of concern. The first area of difference is biomechanical, concerning the physiological sex differences in the head/ neck mass of paracervical musculature. The second area was cultural differences in honesty of reporting and less fear of restriction from play decisions. And the third area was possible hormonal factors such as the protective effect of estrogen in males. ${ }^{15}$

The higher incidence of concussion among female ice hockey players compared with their male counterparts has previously been noted in this discussion section and is documented by Agel et al. ${ }^{1,2}$ These authors suggest that the noncontact designation given to women's hockey may be misleading and be variable at the discretion of the referee. The authors also state that although woman's hockey does not allow open ice hits, it does allow for pinching attackers off along the boards (using the boards surrounding the rink to stop an opponent by forcing your opponent into them) and into the goal, which may result in secondary concussions from contact with the ice, boards, or goals. The suggestion of single player injuries due to lack of control, and sex differences of cervical control muscle strength, should be examined in the future.

Currently, an athlete suspected of sustaining a concussion undergoes neuropsychological evaluation in the form of the internationally accepted SCAT2 screen test and a computerized neuropsychological test. These postinjury values are usually compared with the individual's noninjured or baseline values to determine his or her individual status. They are meant to give the clinician objective tools to assist in making the clinical diagnosis and return-to-play decisions. ${ }^{38}$ In the present study, the only portion of the SCAT2 that demonstrated a statistically significant effect was the total symptom score, and it demonstrated the expected pattern of immediate postconcussion impairment, followed by a gradual return to baseline values.

Mayers and Redick ${ }^{35}$ recently reviewed the literature concerning the clinical utility of the ImPACT computerized neuropsychological testing battery. Citing other recent reviews (especially by Randolph ${ }^{45}$ and Randolph et $\mathrm{al}^{46}$ ) these authors found that tests such as the ImPACT do not meet the reliability and validity criteria desired in a test used for assisting return-to-play decisions. The authors questioned the rationale of using the ImPACT for clinical management of sports-related concussion, and specifically for determining the time of return to play. ${ }^{35}$ The test-retest reliability calculations reported herein support these findings, in that none of the scales achieved a level of reliability that could reasonably be interpreted as sufficient for routine use. Furthermore, athletes appear to be demonstrating a counterintuitive pattern on a portion of the ImPACT (the memory composite [verbal] score). Again, although this result may be a sample-specific effect that occurred due to the small sample size of the present study, the "improvement" shown immediately after a concussion may be a reflection of the high error rate on the measure (the ICC for the memory composite [verbal] score was 0.147), or it may suggest that athletes are purposely malingering at baseline in anticipation of further testing; that is, they purposely perform poorly at baseline so that they will not demonstrate significant impairment at future time points. The latter point is speculation, but it is a plausible explanation of our treatment of the data. From a clinical standpoint, it suggests that tests of malingering should be administered at baseline to ensure that any baseline screening tests are accurate reflections of a player's ability. Mayers and Redick ${ }^{35}$ went on to state "Sports medicine practitioners still lack simple, reliable, and affordable techniques to confidently address these issues. Although experienced first responders can accomplish a clinical diagnosis in most instances of suspected concussion, concerns related to return to play and whether or not to continue specific sport participation are not resolved by current CNT [computerized neuropsychological testing]."

Despite the SCAT2's international development, acceptance, and availability, much like the ImPACT test it has not undergone the rigorous scientific validity and reliability evaluations that would support its use as a valid tool upon which diagnostic and return-to-play decisions 


\section{Ice hockey concussion incidence and neuropsychological changes}

can be assisted. The current published literature concerns the establishment of baseline values on noninjured individuals of different age groups. ${ }^{30,48,49,52}$

The power of the study to make definitive conclusions on incidence rates and the validity of neuropsychological paradigms (SCAT2 and ImPACT) was limited by the number of participants. The testing compliance of the participants was relatively good considering their university age and activity levels. Precise test or retest intervals were occasionally altered to accommodate the schedules or to mitigate the resistance of the participants. The invasive nature of this study caused resistance to compliance concerning self-reporting, diagnostic decision making, and testing. The resistance to incorporating the study methodology was experienced by the players, coaches, therapists, physicians, and administrators throughout this study.

\section{Conclusions}

The present study successfully reproduced the significant incidence of concussion as first documented by Echlin et al. ${ }^{18}$ The significant difference in incidence between the results of this study and our previous study demonstrates the need for improved and independent surveillance studies to be performed at all levels of sports. This study also demonstrated that there was a significant difference in the incidence of concussion between male and female athletes, which has previously been noted in the literature.

This study also examined the sensitivity of the current neuropsychological tools (SCAT2 and ImPACT) that may be used to assist the physician in diagnostic and return-to-play decision making. There were no significant differences found in these measures between preseason and postseason testing among individuals who had not been identified as suffering a concussion during the study period. Furthermore, although some statistically significant changes were demonstrated on the SCAT2 and ImPACT, the latter test demonstrated effects that were more suggestive of player "test-taking-strategies" than they were of sensitivity to injury. This should not necessarily be taken as an indictment of neuropsychological testing per se, but rather that these tests should be administered in the context of other assessments (and clinical evaluations) that evaluate malingering and dissembling. The use of more sensitive objective instruments such as multimodal MRI (MRS, DTI, and SWI) that were used in this study may allow investigators to more clearly define individual diagnostic and recovery parameters.

The cultural acceptance of this serious brain injury, reported as the "invisible epidemic" by the Centers for Disease Control and Prevention in 2002, is an important barrier to progress concerning identification, treatment, and prevention. The lead group in this cultural shift in concussion knowledge must come from the grassroots. The sports that our children play enrich our society. Sport must evolve to minimize concussion incidence as well as improve the identification and treatment of this serious brain injury.

\section{Disclosure}

Funding for this work was provided to the HCEP and Dr. Paul
Echlin by the Ontario Trillium Foundation, the Dave Irwin Foundation for Brain Injury, The Ontario Neurotrauma Foundation, and Air Canada.

Author contributions to the study and manuscript preparation include the following. Conception and design: Echlin, Skopelja, Forwell, Johnson. Acquisition of data: Echlin, Worsley, Dadachanji, Lloyd-Smith, Taunton, Forwell. Analysis and interpretation of data: Echlin, Dadachanji, Forwell, Johnson. Drafting the article: Echlin, Skopelja, Worsley, Forwell, Johnson. Critically revising the article: all authors. Reviewed submitted version of manuscript: all authors. Approved the final version of the manuscript on behalf of all authors: Echlin. Statistical analysis: Johnson. Administrative/ technical/material support: Echlin, Skopelja, Lloyd-Smith, Taunton, Forwell, Johnson. Study supervision: Echlin, Dadachanji, LloydSmith, Taunton, Forwell.

\section{Acknowledgments}

The authors would like to acknowledge the contributions of the University of British Columbia MRI Center and all of the associated researchers and employees, especially Ms. Trudy Harris and Ms. Linda Chandler. The authors also acknowledge the players and staffs of two CIS varsity ice hockey teams for their participation in the HCEP, and would also like to acknowledge the participating physicians, observers, and volunteers for their contributions to the HCEP.

\section{References}

1. Agel J, Dick R, Nelson B, Marshall SW, Dompier TP: Descriptive epidemiology of collegiate women's ice hockey injuries: National Collegiate Athletic Association Injury Surveillance System, 2000-2001 through 2003-2004. J Athl Train 42:249-254, 2007

2. Agel J, Dompier TP, Dick R, Marshall SW: Descriptive epidemiology of collegiate men's ice hockey injuries: National Collegiate Athletic Association Injury Surveillance System, 1988-1989 through 2003-2004. J Athl Train 42:241-248, 2007

3. Agel J, Harvey EJ: A 7-year review of men's and women's ice hockey injuries in the NCAA. Can J Surg 53:319-323, 2010

4. Amen DG, Newberg A, Thatcher R, Jin Y, Wu J, Keator D, et al: Impact of playing American professional football on long-term brain function. J Neuropsychiatry Clin Neurosci 23:98-106, 2011

5. Benson BW, Meeuwisse WH, Rizos J, Kang J, Burke CJ: A prospective study of concussions among National Hockey League players during regular season games: the NHL-NHLPA Concussion Program. CMAJ 183:905-911, 2011

6. Benson BW, Rose MS, Meeuwisse WH: The impact of face shield use on concussions in ice hockey: a multivariate analysis. Br J Sports Med 36:27-32, 2002

7. Covassin T, Elbin RJ, Harris W, Parker T, Kontos A: The role of age and sex in symptoms, neurocognitive performance, and postural stability in athletes after concussion. Am J Sports Med 40:1303-1312, 2012

8. Covassin T, Swanik CB, Sachs ML: Sex differences and the incidence of concussions among collegiate athletes. J Athl Train 38:238-244, 2003

9. Cusimano M, Cook D, Tator C, Mansfield E, Ulrich D: The effectiveness of an injury prevention educational intervention in teaching young hockey players about concussion. Can J Neurol Sci 29 (Suppl 1):S25-S26, 2002 (Abstract)

10. Cusimano MD: Canadian minor hockey participants' knowledge about concussion. Can J Neurol Sci 36:315-320, 2009

11. De Beaumont L, Lassonde M, Leclerc S, Théoret H: Longterm and cumulative effects of sports concussion on motor cortex inhibition. Neurosurgery 61:329-337, 2007

12. De Beaumont L, Théoret H, Mongeon D, Messier J, Leclerc $\mathrm{S}$, Tremblay S, et al: Brain function decline in healthy retired 
athletes who sustained their last sports concussion in early adulthood. Brain 132:695-708, 2009

13. Delaney JS, Lacroix VJ, Gagne C, Antoniou J: Concussions among university football and soccer players: a pilot study. Clin J Sport Med 11:234-240, 2001

14. Delaney JS, Lacroix VJ, Leclerc S, Johnston KM: Concussions during the 1997 Canadian Football League season. Clin J Sport Med 10:9-14, 2000

15. Dick RW: Is there a gender difference in concussion incidence and outcomes? Br J Sports Med 43 (1 Suppl 1):i46-i50, 2009

16. Echlin PS: Concussion education, identification, and treatment within a prospective study of physician-observed junior ice hockey concussions: social context of this scientific intervention. Neurosurg Focus 29 (5):E7, 2010

17. Echlin PS, Johnson AM, Riverin S, Tator CH, Cantu RC, Cusimano MD, et al: A prospective study of concussion education in 2 junior ice hockey teams: implications for sports concussion education. Neurosurg Focus 29(5):E6, 2010

18. Echlin PS, Tator CH, Cusimano MD, Cantu RC, Taunton JE, Upshur REG, et al: A prospective study of physician-observed concussions during junior ice hockey: implications for incidence rates. Neurosurg Focus 29(5):E4, 2010 (Erratum in Neurosurg Focus 29(5):Erratum, 2010)

19. Echlin PS, Tator CH, Cusimano MD, Cantu RC, Taunton JE, Upshur REG, et al: Return to play after an initial or recurrent concussion in a prospective study of physician-observed junior ice hockey concussions: implications for return to play after a concussion. Neurosurg Focus 29(5):E5, 2010

20. Emery CA, Kang J, Shrier I, Goulet C, Hagel BE, Benson BW, et al: Risk of injury associated with body checking among youth ice hockey players. JAMA 303:2265-2272, 2010

21. Emery CA, Meeuwisse WH: Injury rates, risk factors, and mechanisms of injury in minor hockey. Am J Sports Med 34:1960-1969, 2006

22. Flik K, Lyman S, Marx RG: American collegiate men's ice hockey: an analysis of injuries. Am J Sports Med 33:183187,2005

23. Gavett BE, Stern RA, Cantu RC, Nowinski CJ, McKee AC: Mild traumatic brain injury: a risk factor for neurodegeneration. Alzheimers Res Ther 2:18, 2010

24. Gerberich SG, Priest JD, Boen JR, Straub CP, Maxwell RE: Concussion incidences and severity in secondary school varsity football players. Am J Public Health 73:1370-1375, 1983

25. Guskiewicz KM, Marshall SW, Bailes J, McCrea M, Cantu $\mathrm{RC}$, Randolph C, et al: Association between recurrent concussion and late-life cognitive impairment in retired professional football players. Neurosurgery 57:719-726, 2005

26. Guskiewicz KM, Marshall SW, Bailes J, McCrea M, Harding HP Jr, Matthews A, et al: Recurrent concussion and risk of depression in retired professional football players. Med Sci Sports Exerc 39:903-909, 2007

27. Guskiewicz KM, McCrea M, Marshall SW, Cantu RC, Randolph C, Barr W, et al: Cumulative effects associated with recurrent concussion in collegiate football players: the NCAA Concussion Study. JAMA 290:2549-2555, 2003

28. Guskiewicz KM, Weaver NL, Padua DA, Garrett WE Jr: Epidemiology of concussion in collegiate and high school football players. Am J Sports Med 28:643-650, 2000

29. Honey CR: Brain injury in ice hockey. Clin J Sport Med 8: 43-46, 1998

30. Jinguji TM, Bompadre V, Harmon KG, Satchell EK, Gilbert K, Wild J, et al: Sport Concussion Assessment Tool-2: baseline values for high school athletes. Br J Sports Med 46:365370, 2012

31. Kaut KP, DePompei R, Kerr J, Congeni J: Reports of head injury and symptom knowledge among college athletes: implications for assessment and educational intervention. Clin $\mathbf{J}$ Sport Med 13:213-221, 2003

32. Keating P: Reporting from the National Academy of Neuro- psychology's Sports Concussion Symposium: The NHL releases the first data from its concussion program. But what do the numbers mean? ESPN Magazine. (http://sports.espn. go.com/espnmag/story?id=3656016) [Accessed October 30, 2012]

33. Langburt W, Cohen B, Akhthar N, O'Neill K, Lee JC: Incidence of concussion in high school football players of Ohio and Pennsylvania. J Child Neurol 16:83-85, 2001

34. Lehman EJ, Hein MJ, Baron SL, Gersic CM: Neurodegenerative causes of death among retired National Football League players. Neurology [epub ahead of print], 2012

35. Mayers LB, Redick TS: Clinical utility of ImPACT assessment for postconcussion return-to-play counseling: psychometric issues. J Clin Exp Neuropsychol 34:235-242, 2012

36. McCrea M, Hammeke T, Olsen G, Leo P, Guskiewicz K: Unreported concussion in high school football players: implications for prevention. Clin J Sport Med 14:13-17, 2004

37. McCrory P, Johnston K, Meeuwisse W, Aubry M, Cantu R, Dvorak J, et al: Summary and agreement statement of the 2nd International Conference on Concussion in Sport, Prague 2004. Br J Sports Med 39:196-204, 2005

38. McCrory P, Meeuwisse W, Johnston K, Dvorak J, Aubry M, Molloy M, et al: Consensus statement on Concussion in Sport 3rd International Conference on Concussion in Sport held in Zurich, November 2008. Clin J Sport Med 19:185-200, 2009

39. McKee AC, Cantu RC, Nowinski CJ, Hedley-Whyte ET, Gavett $\mathrm{BE}$, Budson $\mathrm{AE}$, et al: Chronic traumatic encephalopathy in athletes: progressive tauopathy after repetitive head injury. J Neuropathol Exp Neurol 68:709-735, 2009

40. McKee AC, Gavett BE, Stern RA, Nowinski CJ, Cantu RC, Kowall NW, et al: TDP-43 proteinopathy and motor neuron disease in chronic traumatic encephalopathy. $\mathbf{J}$ Neuropathol Exp Neurol 69:918-929, 2010

41. Meehan WP III, d'Hemecourt P, Comstock RD: High school concussions in the 2008-2009 academic year: mechanism, symptoms, and management. Am J Sports Med 38:24052409,2010

42. Moser RS, Schatz P, Jordan BD: Prolonged effects of concussion in high school athletes. Neurosurgery 57:300-306, 2005

43. O'Kane JW, Levy MR, Pietila KE, Caine DJ, Schiff MA: Survey of injuries in Seattle area levels 4 to 10 female club gymnasts. Clin J Sport Med 21:486-492, 2011

44. Pinto M, Kuhn JE, Greenfield ML, Hawkins RJ: Prospective analysis of ice hockey injuries at the Junior A level over the course of one season. Clin J Sport Med 9:70-74, 1999

45. Randolph C: Baseline neuropsychological testing in managing sport-related concussion: does it modify risk? Curr Sports Med Rep 10:21-26, 2011

46. Randolph C, McCrea M, Barr WB: Is neuropsychological testing useful in the management of sport-related concussion? J Athl Train 40:139-152, 2005

47. Rishiraj N, Lloyd-Smith R, Lorenz T, Niven B, Michel M: University men's ice hockey: rates and risk of injuries over 6-years. J Sports Med Phys Fitness 49:159-166, 2009

48. Schneider KJ, Emery CA, Kang J, Schneider GM, Meeuwisse WH: Examining Sport Concussion Assessment Tool ratings for male and female youth hockey players with and without a history of concussion. Br J Sports Med 44:1112-1117, 2010

49. Shehata N, Wiley JP, Richea S, Benson BW, Duits L, Meeuwisse WH: Sport concussion assessment tool: baseline values for varsity collision sport athletes. Br J Sports Med 43:730734, 2009

50. Shuttleworth-Edwards AB: Central or peripheral? A positional stance in reaction to the Prague statement on the role of neuropsychological assessment in sports concussion management. Arch Clin Neuropsychol 23:479-485, 2008

51. Sye G, Sullivan SJ, McCrory P: High school rugby players' understanding of concussion and return to play guidelines. $\mathbf{B r}$ J Sports Med 40:1003-1005, 2006 


\section{Ice hockey concussion incidence and neuropsychological changes}

52. Valovich McLeod TC, Bay RC, Lam KC, Chhabra A: Representative baseline values on the Sport Concussion Assessment Tool 2 (SCAT2) in adolescent athletes vary by gender, grade, and concussion history. Am J Sports Med 40:927-933, 2012

53. Wennberg RA, Tator CH: National Hockey League reported concussions, 1986-87 to 2001-02. Can J Neurol Sci 30:206209,2003

54. Wessels KK, Broglio SP, Sosnoff JJ: Concussions in wheelchair basketball. Arch Phys Med Rehabil 93:275-278, 2012

55. Williamson IJS, Goodman D: Converging evidence for the under-reporting of concussions in youth ice hockey. Br J Sports Med 40:128-132, 2006

56. Yard EE, Schroeder MJ, Fields SK, Collins CL, Comstock RD: The epidemiology of United States high school soccer injuries, 2005-2007. Am J Sports Med 36:1930-1937, 2008
57. Zemper ED: Two-year prospective study of relative risk of a second cerebral concussion. Am J Phys Med Rehabil 82: 653-659, 2003

Manuscript submitted August 15, 2012.

Accepted October 22, 2012.

Please include this information when citing this paper: DOI: 10.3171/2012.10.FOCUS12286.

Address correspondence to: Paul S. Echlin, M.D., Elliott Sports Medicine Clinic, 1100 Walkers Line, Suite 2, Burlington, Ontario, Canada L7N 2G3. email: psechlin@gmail.com. 